\title{
5-ALA complete resections go beyond MR contrast enhancement: shift corrected volumetric analysis of the extent of resection in surgery for glioblastoma
}

\author{
Philippe Schucht • Sonja Knittel • Johannes Slotboom • \\ Kathleen Seidel • Michael Murek • Astrid Jilch • \\ Andreas Raabe • Jürgen Beck
}

Received: 1 August 2013 / Accepted: 30 September 2013 /Published online: 25 October 2013

(C) Springer-Verlag Wien 2013

\begin{abstract}
Background The technique of 5-aminolevulinic acid (5-ALA) tumor fluorescence is increasingly used to improve visualization of tumor tissue and thereby to increase the rate of patients with gross total resections. In this study, we measured the resection volumes in patients who underwent 5-ALA-guided surgery for non-eloquent glioblastoma and compared them with the preoperative tumor volume.

Methods We selected 13 patients who had received a complete resection according to intraoperative 5-ALA induced fluorescence and CRET according to post-operative T1 contrast-enhanced MRI. The volumes of pre-operative contrast enhancing tissue, post-operative resection cavity and resected tissue were determined through shift-corrected volumetric analysis.

Results The mean resection cavity $\left(29 \mathrm{~cm}^{3}\right)$ was marginally smaller than the pre-operative contrast-enhancing tumor $\left(39 \mathrm{~cm}^{3}, p=0.32\right)$. However, the mean overall resection volume $\left(84 \mathrm{~cm}^{3}\right)$ was significantly larger than the pre-operative contrast-enhancing tumor $\left(39 \mathrm{~cm}^{3}, p=0.0087\right)$. This yields a mean volume of resected 5-ALA positive, but radiological non-enhancing tissue of $45 \mathrm{~cm}^{3}$. The mean calculated rim of resected tissue surpassed pre-operative tumor diameter by $6 \mathrm{~mm}$ (range $0-10 \mathrm{~mm}$ ).
\end{abstract}

This work was presented in a short talk at the Deutschen Gesellschaft für Neurochirurgie (DGNC) annual meeting in May 2013.

P. Schucht $(\triangle) \cdot$ S. Knittel $\cdot$ K. Seidel $\cdot$ M. Murek $\cdot$ A. Jilch $\cdot$

A. Raabe $\cdot$ J. Beck

Department of Neurosurgery, Inselspital, Bern University Hospital,

Freiburgstrasse 10, 3010 Bern, Switzerland

e-mail: philippe.schucht@insel.ch

J. Slotboom

Department of Diagnostic and Interventional Neuroradiology,

Inselspital, Bern University Hospital, Bern, Switzerland
Conclusions Results of the current study imply that (i) the resection cavity underestimates the volume of resected tissue and (ii) 5-ALA complete resections go significantly beyond the volume of pre-operative contrast-enhancing tumor bulk on MRI, indicating that 5-ALA also stains MRI non-enhancing tumor tissue. Use of 5-ALA may thus enable extension of coalescent tumor resection beyond radiologically evident tumor. The impact of this more extended resection method on time to progression and overall survival has not been determined, and potentially puts adjacent and functionally intact tissue at risk.

Keywords 5-ALA - CRET - Complete resection of enhancing tumor $\cdot$ Extent of resection $\cdot$ Glioblastoma

\section{Introduction}

Although they are solid tumors, glioblastomas are not well delineated and the boundaries are often difficult to discern during white-light surgery. Cell density decreases gradually from the solid tumor tissue towards the infiltrative tumor periphery. The compound 5-aminolevulinic acid (5-ALA) is a technical adjunct that - when given orally before surgeryaccumulates in glioma cells as a fluorescent metabolite and thereby improves both the intraoperative detection of tumor tissue and the extent of resection. The use of 5-ALA as a method of intraoperative imaging has become increasingly popular since a randomized controlled trial showed that the likelihood of achieving a gross total resection (GTR) increased significantly when using 5-ALA induced fluorescence compared to white light only ( $65 \%$ vs $37 \% ; p<0.0001)$ [11]. Fluorescence of 5-ALA is thought to depend not only on the degree of blood-brain barrier disruption, but also on a variety 
of factors such as an increased cellular metabolism and a modified tumor microenvironment [3].

Gadolinium-enhanced T1-weighted MR sequencesthe modality of choice to detect solid tumor outside of surgery-detects the tumor's disrupted blood-brain barrier [2]. Thus, T1 enhanced magnetic resonance imaging (MRI) and intra-operative 5-ALA imaging identify different characteristics of a tumor, and may depict a different extent of tumor in a given patient. While the ability of 5-ALA guided surgery to resect at least the contrast enhancing tumor part (i.e. GTR) is undisputed after the 5-ALA study [11], it remains unclear to what extent the use of 5-ALA leads to tissue resection beyond contrast enhancement.

It is of great clinical importance for the planning of surgery to know if, and to what extent, 5-ALA fluorescence visualizes tumor tissue beyond the gadolinium-enhancing rim on preoperative MRI, as larger resections may endanger nearby eloquent areas. In addition, it may be of conceptual significance to know if 5-ALA guides towards a more complete tumor resection than indicated by a T1-contrast MRI.

The aim of our study was to compare the volume of contrast enhancing tumor as seen on pre-operative T1contrast MRI with the resection volume on the postoperative T1 MRI after 5-ALA-guided surgery for noneloquent glioblastoma.

\section{Methods}

Study protocol and inclusion criteria

Data regarding presenting symptoms, Karnofsky Performance Scale, perioperative morbidity and mortality were retrieved from our departmental prospective glioma register. The register included results of complete clinical and neurological examinations performed on the day before surgery, at discharge, and 6 to 8 weeks after surgery. All patients were operated upon according to our institutional protocol which stipulates that glioblastoma resection in presumed noneloquent areas should progress according to fluorescence until complete removal of all fluorescent tissue (both solid and vague fluorescence). Pre- and postoperative neurologic deficits were recorded, in addition to any changes in the degree of deficits over time. All 102 patients who underwent surgery for glioblastoma from 1 August 2008 to 30 July 2010 at the Department of Neurosurgery, Bern University Hospital, Bern, Switzerland, were screened for this study. Preoperative MRI not older than 7 days at the time of surgery and complete resection of enhancing tissue (CRET) on early post-operative T1-contrast MRI were prerequisites for study inclusion. For methodological reasons related to the shift corrected volumetric analysis we selected 13 patients with a spherical, non-multifocal contrast enhanced tumor with a volume larger than $10 \mathrm{~cm}^{3}$ and located close to the brain surface in a non-eloquent area. All patients signed a surgical consent form. The local Ethics committee approved the study (Cantonal Ethics Commission Bern, 10-8-2010).

Imaging protocol

Specifications of pre- and post-operative MRI were identical: All MRIs were performed on a 1.5 or a $3 \mathrm{~T}$ scanner with a head coil. Unenhanced and enhanced T1 sequences without gap were obtained (magnetized-prepared rapid-acquisition gradient echo [MP-RAGE] sequences with $0.1 \mathrm{mmol} / \mathrm{kg}$ bodyweight gadolinium-diethylenetriaminepentaacetic acid [DTPA] given intravenously, $256 \times 256$ matrix, rectangular field of view, slice thickness $1 \mathrm{~mm}$ ). Post-operative MRI was obtained within $48 \mathrm{~h}$ after surgery.

\section{5-ALA and surgery protocol}

All patients received $4 \mathrm{mg}$ of dexamethasone three times a day for at least 3 days prior to surgery (with the exception of patient 5, who received steroids only during surgery). 5ALA ( $20 \mathrm{mg} / \mathrm{kg}$ body-weight) was diluted in $50 \mathrm{~mL}$ of water and given orally $3 \mathrm{~h}$ before the induction of anesthesia (range $2-4 \mathrm{~h})$. All patients received mannitol $(20 \%, 250 \mathrm{ml})$ at the beginning of surgery. Surgery was carried out with the use of a neurosurgical microscope (OPMI Pentero, Carl Zeiss) equipped with the fluorescence kit. Blue light was used for identification of tumor tissue at several stages of surgery. Surgery was performed according to fluorescence with the aim to remove all fluorescent (solid and vague) tissue while sparing non-fluorescent parenchyma. Central necrosis was resected regardless of fluorescence. At the end of surgery, the resection cavity was again checked with blue light to ensure completeness of tumor removal. Neuronavigation was performed in all cases for localization control.

\section{Processing of image data}

Imaging data from pre- and post-operative MRI were uploaded into Brainlab ${ }^{\circledR}$ iPlan 3.0.2 cranial software (VectorVision, Brainlab, Heimstetten, Germany). Volumetric analysis was performed through manual segmentation across all slices (volume 1) or manually corrected autosegmentation (volumes 2 and 3) [6]. All volumetric assessments were performed on gadolinium-enhanced T1 sequences (MP-RAGE). The following volumes were obtained:

1. Pre-operative tumor volume: All tissue with pathological enhancement on pre-operative gadolinium-enhanced $\mathrm{T} 1$ sequences; plus central necrosis 
2. Volume of the resection cavity: Cavity at the former tumor site, including debris and blood, as seen on post-operative gadolinium-enhanced $\mathrm{T} 1$ sequences

3. Pre- and post-operative brain volume: The autosegmentation feature of the iPlan software was used to obtain the cerebral volume (supratentorial brain volume without CSF spaces and tumor). Using anatomical atlas information for initialization, this feature is based on an expectation-maximization algorithm under specific statistical assumptions on the gray value distribution of brain tissue classes [14]. A manual slice per slice correction ensured optimized accuracy.

4. The volume of resected tissue that did not enhance in the pre-operative MRI was obtained with the formula:

Volume of resected non-enhancing tissue $=$

Preoperative Brain volume-Postoperative Brain volume

5. The overall volume of resected tissue is the sum of enhancing and non-enhancing resected tissue:

Overall resection volume $=$

Tumor volume + volume of resected non-enhancing tissue

6. Additional 5-ALA resection margin calculation

The mean tumor diameter is calculated using tumor volume (we postulate a spherical resection, analogous to the pre-operative spherical tumor shape [inclusion criterion]). The diameter of the resected tissue is calculated accordingly.

Standard deviations for calculated quantities (tumor diameter, volume of resected unenhancing tissue, overall resection volume, total resected tissue diameter, volume of resection cavity in percentage of pre-op enhancing tumor, volume of resected tissue in percentage of pre-op enhancing tumor, mean resected rim beyond contrast enhancement) were estimated according to the rules of error propagation [5].

\section{Results}

Demographics and tumor volume

Mean age at surgery was 61 years (range $48-80$ years), and 6 of the 13 patients were women. Steroids were given for a mean duration of 6 days prior to imaging (range $0-16$ days) and 3.5 days prior to surgery (range $0-15$ days). All preoperative MRIs were not older than 1 week at the time of surgery. Mean pre-operative tumor volume (contrast enhancement on gadolinium-enhanced $\mathrm{T} 1$ sequences plus necrosis) was $39 \mathrm{~cm}^{3}$ (range 11-93 $\mathrm{cm}^{3}$ ). Intra-operative electrophysiologic mapping and monitoring was used in three patients as a safety measure due to the tumor's proximity to cortical and subcortical motor areas: Somatosensory evoked potentials (SSEP), trans-epidermal stimulation motor evoked potentials (TES MEP) and cortical motor mapping in patient 2; SSEP, TES MEP and direct cortical stimulation MEP (DCS MEP) in patients 10 and 13. Monitoring signals were stable in all patients and thus did not lead to early termination of resection.

Volume of the resection cavity

The resection cavity without any shift correction had a mean volume of $29 \mathrm{~cm}^{3}$ (range $6-80 \mathrm{~cm}^{3}$ ) according to the postoperative T1 MRI (Table 1) and was thus marginally smaller than the pre-operative contrast enhancement (unpaired $t$-test, $95 \%$ confidence interval -30.3 to 10.4 , two-tailed $P=0.32$ ), although no contrast enhancing remnant was seen on the postoperative gadolinium-enhanced MRI (all patients had GTR). For individual patients, the resection cavities' relative size compared to the preoperative tumor ranged from $22 \%$ to $192 \%$ (Table 1).

Pre- and post-operative brain volume, and volume of resected non-enhancing tissue

The mean pre-operative brain volume (supratentorial brain without enhancing tumor, central necrosis) was $1,077 \mathrm{~cm}^{3}$ (range $967-1,253 \mathrm{~cm}^{3}$ ). The early post-operative MRI showed a mean brain volume of $1,032 \mathrm{~cm}^{3}$ (range $870-1,199 \mathrm{~cm}^{3}$ ). The mean volume of resected tissue that did not enhance on gadolinium-enhanced pre-operative MRI was calculated to be $46 \mathrm{~cm}^{3}$ (range $1-120 \mathrm{~cm}^{3}$ ).

\section{Overall volume of resected tissue}

The overall resection volume, encompassing enhancing and non-enhancing tissue, correlated well with the volume of GD enhancing tumor on pre-operative MRI (Fig. 1). Overall resection volume was $84 \mathrm{~cm}^{3}$ (range of $15-191 \mathrm{~cm}^{3}$ ) and thus significantly larger than the mean pre-operative T1-contrastenhancing tumor volume of $39 \mathrm{~cm}^{3}$ (unpaired $t$-test, $95 \% \mathrm{CI}$ 8.6 to 80.9 , one-tailed $P=0.0087$ ). In fact, the mean relative size of the overall resection volume compared to pre-operative tumor volume was $242 \%$ (range 101-494\%, Table 1).

Additional 5-ALA resection margin calculation

Comparing the volume-derived diameter of the pre-operative tumor (mean $4.0 \mathrm{~cm}$, range $2.7-5.6 \mathrm{~cm}$ ) and of resected tissue (mean $5.2 \mathrm{~cm}$, range $3.1-7.1 \mathrm{~cm}$ ), the resection using 5-ALA yielded a mean additional rim or resection margin of $6 \mathrm{~mm}$ that was removed beyond the $\mathrm{T} 1$ contrast enhancement border (range $0-10 \mathrm{~mm}$ ). 


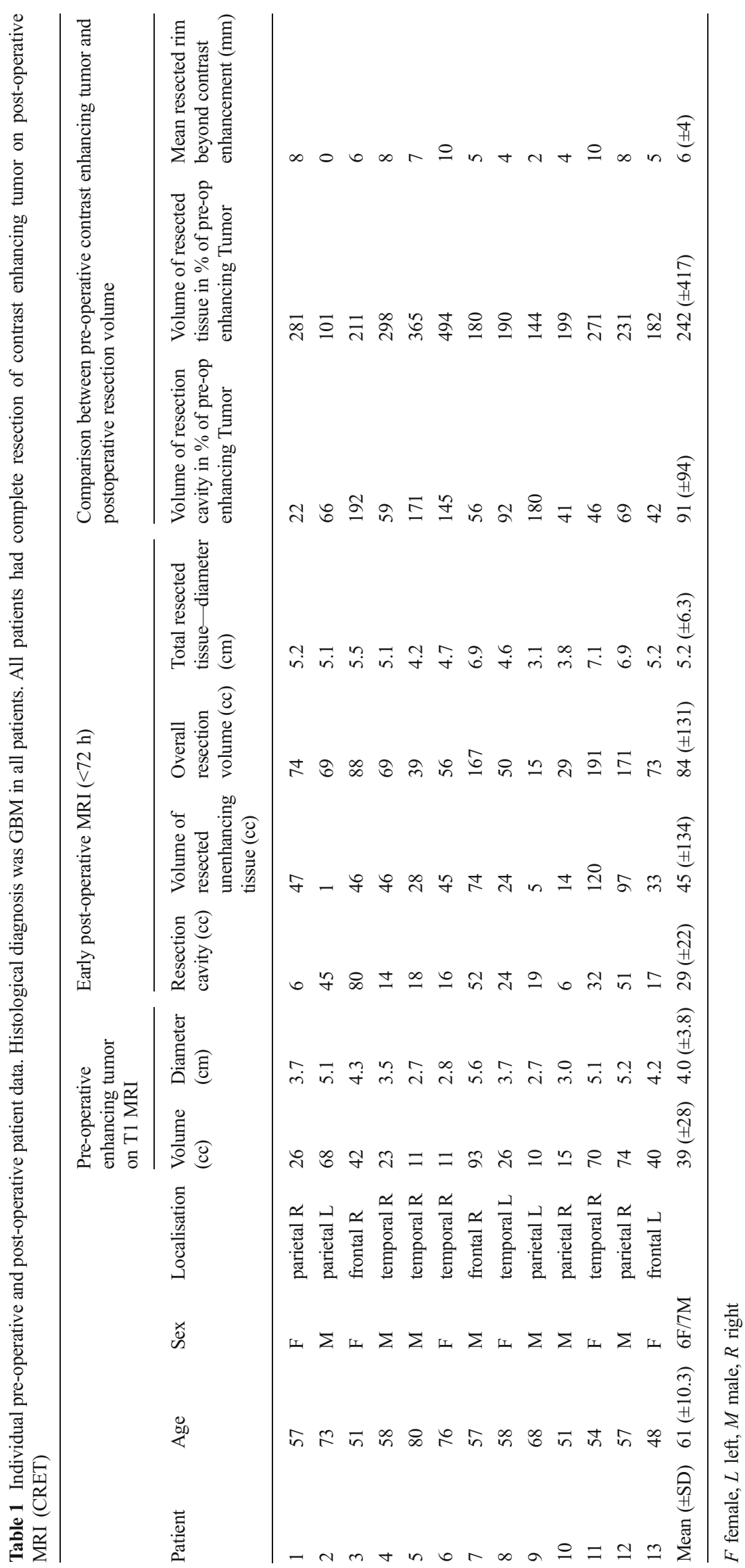


Fig. 1 Correlation between the volume of contrast enhancement on pre-operative MRI and the overall resection volume.

Pearson's correlation coefficient: $0.8676(95 \%$ CI $0.6066-0.9598)$. $p=0.0001215$

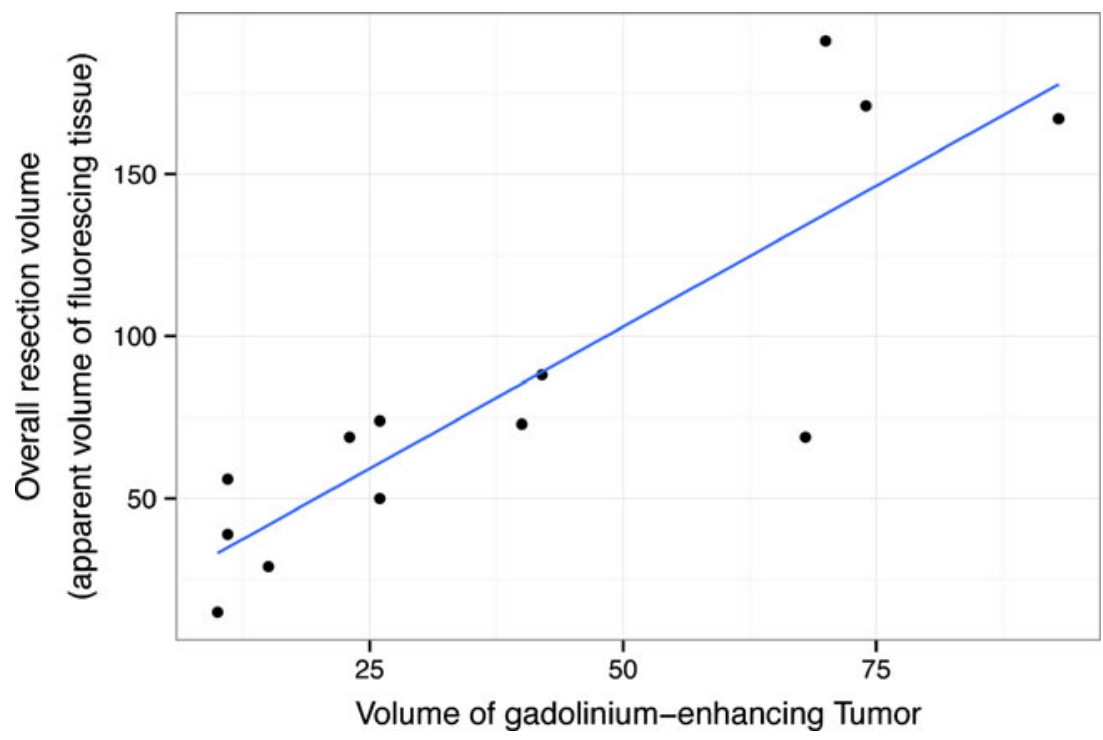

Neurologic deficits

Six patients presented with minor neurological deficits prior to surgery, of which two improved after administration of steroids and surgery. Postoperatively, we observed no new or worsened neurologic deficits in the 13 included patients. No intracranial infection, infarction or hydrocephalus was observed within 3 months after surgery.

\section{Discussion}

Evaluation of shift corrected volumetry

Logic implies that after radiological CRET the resection cavity must be at least the size of the preoperative contrast enhancement. However, on early post-operative MRI we observed resection cavities that were smaller than the preoperative contrast-enhancing tumor in 9 of 13 patients due to shifting of the previously dislocated surrounding brain tissue into the resection cavity following tumor removal (Table 1, Fig. 2). The resection cavity's volume thus turned out to be an imprecise surrogate parameter for the amount of resected tissue in our series. The problem of brain shift may be bypassed when the volume of resected tissue is calculated by comparing pre- and post-operative cerebral volume.

True resection volumes and methodological limitations

Volumetric assessment of pre- and post-operative brain volumes suggest that the volume of resected tissue was more than twice the volume of gadolinium-enhancing tissue on preoperative imaging $(+111 \%)$. Methodological limitations have to be considered because our measurements are essentially indirect. The discrepancy between contrast-enhancing tumor volume and volume of resected tissue is unlikely to be due to tumor growth, as none of the pre-operative MRI scans were older than 7 days. In addition, brain edema and the resolution thereof after surgery may have influenced our results, despite the fact that the patients were under corticosteroid treatment during the perioperative period. Another possible source of error may be resection of non-fluorescent tissue in the approach corridor. However, resection of non-fluorescent tissue during corticotomy was minimal in these cases specifically selected for tumor localization close to the brain's surface. Thus, we believe that the discrepancy between the preoperative enhancing tumor and the volume of resected tumor is due to the different sensitivities of gadolinium and 5-ALA in depicting tumor tissue (Fig. 3). However, some limitations of our methodology have to be considered, as various factors may influence outcome of our indirect measurements.

Sensitivity and specificity of 5-ALA induced fluorescence

A post-hoc analysis of the 5-ALA randomized controlled trial with 322 patients revealed that $34 \%$ of patients in whom surgery was terminated despite residual tumor had a complete resection according to post-operative MRI [12]. This questions the sensitivity of gadolinium-enhanced MRI in identifying residual coalescent tumor tissue. In a prospective series of 52 patients, $35(67 \%)$ showed residual 5-ALA fluorescence (23 solid and 12 vague fluorescence) at the end of resection, but only 19 (37\%) patients had remaining contrast enhancement on the postoperative T1-contrast MRI [10]. The same study also confirmed the high specificity of 5-ALA induced fluorescence, as all but one of 212 serial biopsies of fluorescent tissue contained tumor cells. These findings imply that the resection of all fluorescent tissue (5-ALA complete 
Fig. 2 Gadolinium-enhanced T1-weighted MR sequences of patient 9 . The resection cavity (right side, post-operative) appears larger than the preoperative contrast-enhancing tumor (left side) after 5 ALAguided surgery

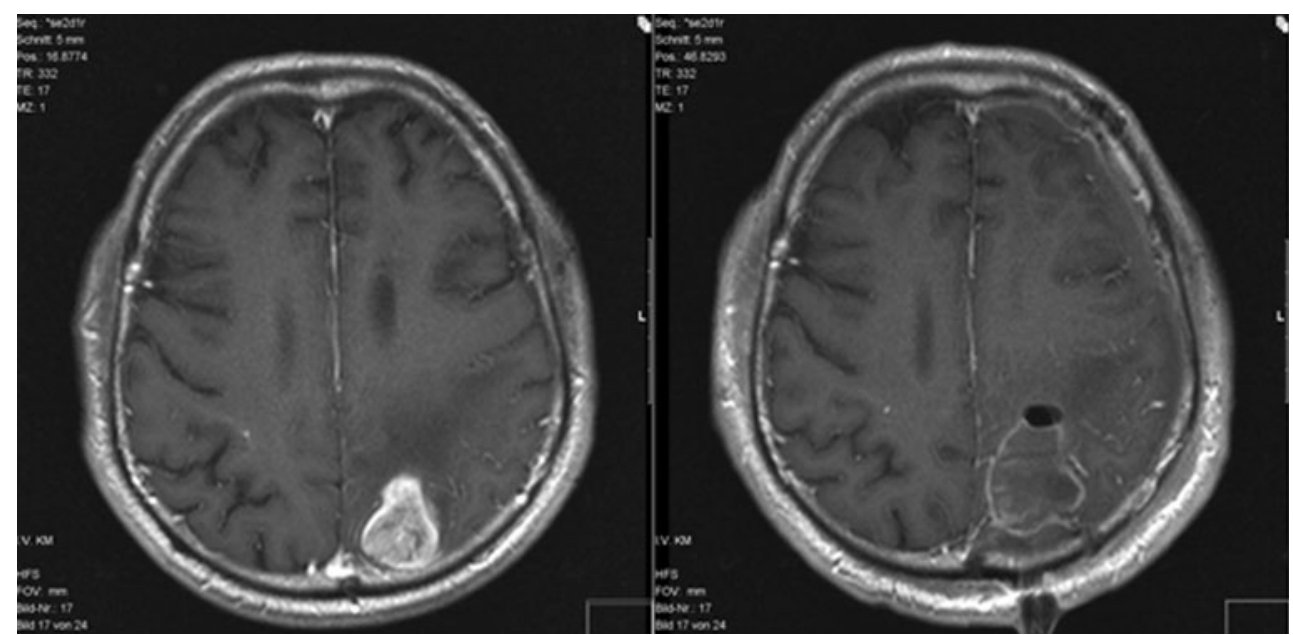

resections) should lead to resection volumes greater than the preoperative contrast enhancement on the T1 MRI. Our own results are in line with this assumption.

Diameter of resected tissue: implication for surgical planning

It is of practical importance to know how far 5-ALA fluorescence extends beyond the enhancing tumor seen on MRI. The comparison of calculated mean diameter of preoperative tumor and of resected tissue volume shows that a rim with a mean width of $6 \mathrm{~mm}$ (range $0-10 \mathrm{~mm}$ ) was resected beyond contrast enhancement (Table 1). Of course, this 5-ALApositive/gadolinium-negative rim may not be homogenous in all directions as glioma cells are known to migrate alongside white matter tracts $[4,7]$. However, as a rule of thumb, we believe that a rim of $1 \mathrm{~cm}$ around the enhancing tumor should be regarded as tissue at risk of being resected in 5-ALA guided surgery.
Risks of 5-ALA guided surgery

Surgery guided by 5-ALA has been shown to carry additional risks of neurological deficits compared to white light only [13]. Our own data show that 5-ALA complete resections go beyond the contrast enhancing tissue on MRI T1 sequences, therefore involving tissue which might have been regarded as mainly non-tumoral, functioning brain tissue prior to surgery. The risk of removing functional brain tissue and therefore inflicting neurological deficits might increase when attempting 5-ALA complete resections, as the likelihood of infiltrated brain tissue instead of coalescent tumor is higher in the peripheral vaguely fluorescent area compared to areas of solid fluorescence ( $88 \%$ versus $46 \%, p=0.0136$ ) [10]. No neurologic deficits were observed in our series, but we only included tumors in non-eloquent locations and had just 3 tumors within $1 \mathrm{~cm}$ from the primary motor cortex and the corticospinal tract. However, the devoted use of different available safety enhancing surgical adjuncts such as cortical
Fig. 3 Gadolinium-enhanced T1-weighted MR sequences of patient 2 . The resection cavity (right side, post-operative) appears smaller than the preoperative contrast-enhancing tumor (left side) after 5 ALAguided surgery due to shift of the surrounding brain parenchyma into the resection cavity

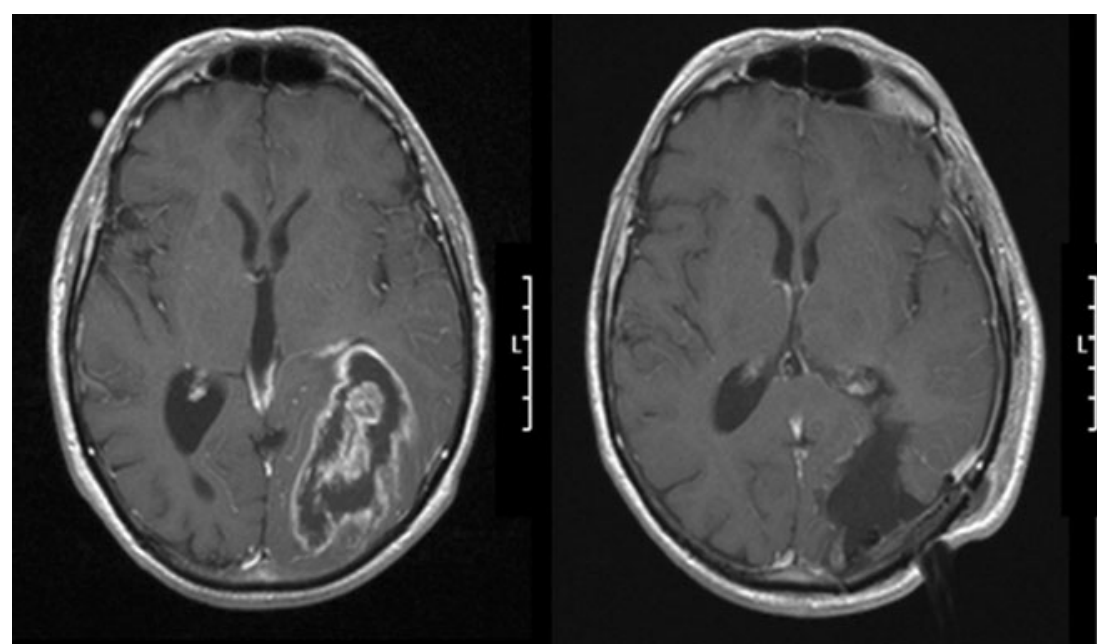


and subcortical mapping, MEP monitoring and speech monitoring in awake settings help to identify the essential functional boundaries and thus contribute to the goal of maximizing tumor resection while keeping postoperative deficits at an acceptable level $[8,9]$. According to the mentioned rule of thumb, we believe that a $1-\mathrm{cm}$ rim around the enhancing tumor should be included when assessing the eloquency of a tumor localization, prompting the use of safety enhancing surgical adjuncts depending on the eloquent area at risk.

Is there a survival benefit from 5-ALA complete resection compared to GTR/CRET?

The 5-ALA randomized controlled trial had two important findings: It showed that the use of 5-ALA leads to an increased rate of GTR (according to postoperative MRI) and demonstrated in its post hoc analysis an increased overall survival in patients who received a GTR compared to those who did not (16.9 versus 11.8 months, $p<0.0001)[11,12]$. The same study did not state, however, whether patients had 5-ALA complete resections and it therefore remains yet unknown whether extending tumor resection beyond contrast enhancing tissue on MRI has an additional survival benefit for the patient. Notably, a recent retrospective cohort study investigated the survival benefit of 5-ALA complete resections in patients who received CRET. Patients with 5-ALA complete resection had a significantly longer overall survival than those with persisting fluorescence at the end of surgery (27 months versus 17.5 months, $p=0.015$ ), despite the fact that all patients had a complete resection of enhancing tumor [1]. The potential benefit of 5-ALA complete resections certainly deserves further investigation and must be weighed against a possible risk of additional neurological deficits after extended resections.

\section{Conclusions}

In our series, the resection cavity was a surprisingly imprecise surrogate parameter for the amount of resected tumor tissue due to post-operative brain shift. We demonstrate by volumetric means that extending glioblastoma resection to a complete removal of 5-ALA induced fluorescent tumor (5-ALA complete resection) leads to resection volumes significantly larger than the volume of contrast-enhancing tumor according to the preoperative T1-weighted MRI, and thus to resection of non-gadolinium-enhancing tumor tissue. As a rule of thumb, 5-ALA guided surgery should be used with caution if preoperative MRI shows enhancement within $1 \mathrm{~cm}$ of eloquent areas. Given the high specificity of 5-ALA induced fluorescence for detecting tumor tissue we must assume that 5-ALA guided surgery is more efficient in detecting and guiding removal of tumor tissue than surgery relying solely on pre- operative MRI imaging. Even though the use of 5-ALA guided surgery has been proven to increase the likelihood of achieving a prognostic advantageous radiologic GTR, the prognostic impact of a 5-ALA complete resection on survival has not been evaluated to date. It remains to be seen whether the known prognostic benefit of 5-ALA guided surgery comes from its ability to resect tumor beyond contrast enhancement or solely from increasing the likelihood of performing a radiological GTR.

Conflicts of interest The authors report no conflicts of interest concerning the materials or methods used or the findings specified in this paper. No specific funding was provided for this work.

\section{References}

1. Aldave G, Tejada S, Pay E, Marigil M, Bejarano B, Idoate MA, DiezValle R (2013) Prognostic value of residual fluorescent tissue in glioblastoma patients after gross total resection in 5-aminolevulinic Acid-guided surgery. Neurosurgery 72:915-920, discussion 920-921

2. Berger MS (2011) Glioma surgery: a century of challenge. Clin Neursurg 58:7-9

3. Collaud S, Juzeniene A, Moan J, Lange N (2004) On the selectivity of 5-aminolevulinic acid-induced protoporphyrin IX formation. Current medicinal chemistry. Curr Med Chem Anticancer Agents 4: 301-316

4. Konukoglu E, Clatz O, Bondiau PY, Delingette H, Ayache N (2010) Extrapolating glioma invasion margin in brain magnetic resonance images: suggesting new irradiation margins. Med Image Anal 14: 111-125

5. Ku HH (1969) Notes on the use of propagation of error formulas. J Res NBS C Eng Inst 70C:263-273

6. Kuhnt D, Becker A, Ganslandt O, Bauer M, Buchfelder M, Nimsky C (2011) Correlation of the extent of tumor volume resection and patient survival in surgery of glioblastoma multiforme with high-field intraoperative MRI guidance. Neuro-oncology 13:1339-1348

7. Scherer HJ (1940) The forms of growth in gliomas and their practical significance. Brain 63:1-35

8. Schucht P, Beck J, Abu-Isa J, Andereggen L, Murek M, Seidel K, Stieglitz L, Raabe A (2012) Gross total resection rates in contemporary glioblastoma surgery: results of an institutional protocol combining 5-aminolevulinic acid intraoperative fluorescence imaging and brain mapping. Neurosurgery 71:927-935, discussion 935-936

9. Seidel K, Beck J, Stieglitz L, Schucht P, Raabe A (2013) The warning-sign hierarchy between quantitative subcortical motor mapping and continuous motor evoked potential monitoring during resection of supratentorial brain tumors. J Neurosurg 118:287-296

10. Stummer W, Novotny A, Stepp H, Goetz C, Bise K, Reulen HJ (2000) Fluorescence-guided resection of glioblastoma multiforme by using 5 -aminolevulinic acid-induced porphyrins: a prospective study in 52 consecutive patients. J Neurosurg 93:1003-1013

11. Stummer W, Pichlmeier U, Meinel T, Wiestler OD, Zanella F, Reulen HJ, Group AL-GS (2006) Fluorescence-guided surgery with 5aminolevulinic acid for resection of malignant glioma: a randomised controlled multicentre phase III trial. Lancet Oncol 7:392-401

12. Stummer W, Reulen HJ, Meinel T, Pichlmeier U, Schumacher W, Tonn JC, Rohde V, Oppel F, Turowski B, Woiciechowsky C, Franz K, Pietsch T, Group AL-GS (2008) Extent of resection and survival in glioblastoma multiforme: identification of and adjustment for bias. Neurosurgery 62:564-576 
13. Stummer W, Tonn JC, Mehdorn HM, Nestler U, Franz K, Goetz C, Bink A, Pichlmeier U, Group AL-GS (2011) Counterbalancing risks and gains from extended resections in malignant glioma surgery: a supplemental analysis from the randomized 5-aminolevulinic acid glioma resection study. Clinical article. J Neurosurg 114:613-623

14. Van Leemput KM, Maes F, Vandermeulen D, Suetens P (1999) Automated model-based tissue classification of MR images of the brain. IEEE Trans Med Imaging 18:897-908

\section{Comment}

This paper by Schucht et al. addresses an important aspect of fluorescenceguided resections, which is that the volume of ALA-derived tumor fluorescence during surgery of malignant gliomas markedly surpasses the volume of Gd-enhancement. The volume of Gd-enhancing tissue has always been the traditional target tissue in surgery for these diffusely infiltrating lesions and complete resection of Gd-enhancing tumor is one of the strongest prognostic indicators. Schucht and co-workers quantified the volume of fluorescing tissue by subtracting brain volume after surgery from brain volume prior to surgery, and correcting for CSF spaces. Nevertheless, this elegant approach has methodological limitations, since changes in brain blood volume or edema resolutions after resection of the tumor mass were not quantified and possibly cannot be quantified. Still, the approximation is probably quite good.

This paper complements recent data from Pamplona (1), in which Aldave and co-workers demonstrate that patients have better survival if fluorescing tissue is removed completely rather than leaving fluorescing tissue behind, even if residual tumor does not show up as GDenhancement on the MRI. With this experience in mind, one might consider redefining the aim of surgery for malignant gliomas to extend resections to include all fluorescing tissue. Of course, maintaining functionality in patients is of utmost importance, and neurophysiological monitoring and mapping techniques have become indispensible adjuncts in the surgical therapy of patients with malignant gliomas when using the potential afforded by ALA-induced tumor fluorescence.

\section{Walter Stummer}

Münster, Germany

\section{Reference:}

(1) Aldave G, Tejada S, Pay E, Marigil M, Bejarano B, Idoate MA, Díez-Valle R (2013) Prognostic value of residual fluorescent tissue in glioblastoma patients after gross total resection in 5-aminolevulinic Acidguided surgery. Neurosurgery. 72(6):915-20 